\title{
Successful STEM student pathways: a two- and four-year partnership
}

\author{
Charles J. De Leone, ${ }^{1}$ Edward Price, ${ }^{1}$ Debbie DeRoma, ${ }^{1}$ Chandra Turpen, ${ }^{2}$ and Daniel Sourbeer ${ }^{3}$ \\ ${ }^{1}$ Department of Physics, California State University San Marcos,
333 South Twin Oaks Valley Road, San Marcos, CA 92096 \\ ${ }^{2}$ Department of Physics, University of Maryland, College Park, MD 20742 \\ ${ }^{3}$ Palomar College, 1140 West Mission Road, San Marcos, CA 92069
}

\begin{abstract}
Many geographically related two- and four-year institutions share a large percentage of their students. However, physics- or STEM-specific linkages between the institutions are often weak, despite the benefits of such linkages to the students. We have been engaged in a multi-year effort to strengthen the partnership between the physics and other STEM programs at two regional institutions, California State University San Marcos and Palomar College, with the goal of creating more coherent STEM pathways for students. Through steps such as building personal relationships among faculty and administrators across campuses, creating coherent academic support services, and establishing formal pathways for students, the partnership improved outcomes for students, including increases in the number of two-year students prepared in STEM domains, number of transfers, and the success of transfers within CSUSM STEM programs. In describing this process, we identify critical elements such as mutual respect, shared goals, aligned institutional missions, and the catalyst of external funding.
\end{abstract}

\section{INTRODUCTION}

Undergraduate STEM education takes place in a complex ecosystem including K12 schools, two-year colleges, and four-year universities. As students move between institutions, discontinuities and inconsistencies can create barriers to success, particularly for students from underrepresented groups or first generation college students [1]. Strategic partnerships, including well-coordinated course transformations, can address these challenges by helping create coherent, interconnected pathways. However, research on faculty change highlights the inadequacy of basic "dissemination" models and the complexity of educational transformation [2,3].

The joint California State University San Marcos (CSUSM) and Palomar College STEP Project, "Increasing STEM talent through regional partnerships, recruiting, and retention" aimed to lower institutional barriers for transfer students and foster success for all STEM majors. Both Palomar and CSUSM are located in the north San Diego region, an area that has traditionally not produced the number of STEM graduates needed for local industries. The project was initiated in Fall 2011 with dual goals of increasing the number of CSUSM graduates in STEM fields and increasing the number of STEM transfer students from Palomar College, particularly in Physics. Project strategies focused on both the retention of and recruitment of STEM majors.

Significant project achievements include: (i) greater than expected increases in CSUSM STEM graduates (183 STEM graduates in 2014-15 vs. 99 in 2010-11), (ii) an increase in the number of transfers from Palomar College into STEM disciplines at CSUSM (roughly 133 in Fall 2015 vs. 58 in Fall 2010), (iii) increases in CSUSM enrolled STEM majors (2114 in Fall 2015 from 866 in Fall 2010 with 41\% coming from traditionally under-represented groups), (iv) increases in transfers from Palomar to CSUSM intending to major in Physics (from 2 in Fall 2011 to 11 in Fall 2015), and (v) intercampus faculty learning communities that have installed Discipline Based Education Research (DBER) practices in gateway STEM courses in Math and Chemistry.

The overall finding is one of clear positive effects on STEM students via increases in the numbers and diversity of STEM majors and graduates. This paper focuses on the effort to improve the pathway for physics students and more generally STEM students from Palomar College to CSUSM and ultimately graduation. These project elements include crosscampus faculty learning communities, a cross-campus Learning Assistant program, physics-specific linkages, and administrative collaboration.

\section{PROJECT ELEMENTS}

In order to increase the number of students graduating from CSUSM with STEM degrees, the STEP project sought to create a vertical pathway for STEM education in North County San Diego schools. When a 2011 research study of local high schools and community colleges revealed a lack of awareness of the university's STEM programs, the STEP team initiated conversations with administrators from Palomar College. Although the initial cultivation of these partnerships involved a significant time investment, these long-term, reciprocal relationships between university faculty and community college personnel proved key to the development and success of the STEP recruiting initiatives. As a member of the Palomar administrative leadership stated during an interview: "It's really all about relationships. Sure, physical proximity is convenient, but it is the relationships that really matter, the camaraderie that develops over time. Pipelines develop that way, from the relationships. Palomar faculty can genuinely and authentically endorse the CSUSM campus." Once informed, these instructors and administrators acted as advocates for the CSUSM STEM programs. 


\section{A. Active Learning Pedagogy Support (ALPS)}

Palomar College and CSUSM serve the same geographical area, and prior to the inception of the project, some STEM specific linkages - including joint undergraduate research experiences and sporadic STEM faculty meetings - between the institutions already existed. The STEP project activities focused on strengthening and expanding these existing collaborations. One key project element was the Active Learning Pedagogy Support Program (ALPS). The ALPS program utilized faculty learning communities (FLCs) [4] that were cross-campus and discipline-based.

The goal of these groups is to (i) explore evidence-based instructional practices, (ii) plan the implementation of such practices in participating faculty's classes, and then (iii) implement these practices with the help of undergraduate Learning Assistants (LAs). The ALPS program capitalizes on the wealth of proven curricular reforms while addressing the challenges such as local adaptation, faculty development, and buy-in associated with implementation and sustainability. Evidence shows that FLCs increase faculty interest in teaching and learning and provide support to change longstanding instructional practices $[5,6]$. Furthermore, with the participation of faculty from neighboring two- and four- year institutions, ALPS promotes cross-campus coordination at the disciplinary or departmental level.

In Spring 2012, an introductory chemistry ALPS team with 10 faculty was formed. The group included eight members from CSUSM and two from Palomar College. The team was led by the chair of the CSUSM chemistry department and included the chair of the Palomar College chemistry department along with a mix of tenure-track faculty and lecturers. All participants received a modest stipend (approximately $\$ 1500$ ) in return for a two-semester commitment to attend the group meetings and implement active learning strategies in at least one course. The chemistry chair was responsible for scheduling the meetings, soliciting topics of interest from group members, and facilitating the group discussions.

The chemistry group convened bi-weekly to explore and develop evidence-based curricular modifications. They identified a number of possibilities, including active learning techniques (such as the use of personal response systems), homework modifications (including on-line homework), peer instruction, and placement/advising exams. In Fall 2012, we leveraged the existing CSUSM Learning Assistant(LA) program to support the participating chemistry faculty as they implemented their course modifications.

A mathematics ALPS group was formed in the Fall 2012. This group consisted of six CSUSM faculty and two Palomar College faculty. The team was led by a CSUSM professor, and the participants included the chair of the CSUSM math department. The group met bi-weekly during Fall 2012 and Spring 2013 to discuss evidence-based instructional techniques. During this time, group members developed and piloted active-learning based interventions for historically difficult mathematics topics. The interventions were implemented in calculus and pre-calculus courses on both campuses with the support of LAs.

The mathematics ALPS group was particularly proactive, and the members continued to meet beyond the initial yearlong obligation. In Spring 2014, a cross-campus sub-group of math faculty formed a lesson study team to explore possible changes in the pre-calculus and Calculus I curricula. Interviews with participating faculty provided insight into the norms of the math ALPS group. A Palomar faculty member commented, "Neither of us from Palomar had Ph.D.s and most of the faculty at CSUSM, I think, did have Ph.D.s, but they didn't seem to care. They didn't really ask if you had a Ph.D... everyone was valued equally and was working toward a common goal." This faculty member continued, "Cal state folks could have said... you aren't preparing your student for San Marcos adequately, and that wasn't said. The feeling was that we all want to prepare our students to the same goals..." The impact of modified lessons was evaluated through student feedback and observations by the ALPS faculty and LAs. A Palomar faculty member described the observations, "In observing each others' classes, they [the CSUSM faculty] were really interested in our feedback. They were interested in what we were seeing and observing." The math ALPS group also brought in an outside speaker to share research on pedagogical strategies in college calculus. One of the group's participants, an adjunct professor at CSUSM, applied the knowledge he gained from the ALPS discussions to his introductory calculus courses, resulting in significant curricular changes and improved student outcomes. With the support of his LAs, the professor explored various interactive learning strategies including clickers, flipped classrooms, and small group problem-solving.

\section{B. Cross-campus Learning Assistant program}

The STEP project leveraged the existing CSUSM Learning Assistant (LA) program to support faculty members who participated in the ALPS project. Based on the University of Colorado, Boulder model, [7], the LA program aims to increase active learning opportunities in science and math courses while simultaneously introducing undergraduate STEM majors to careers in teaching. LAs help with in-class activities, meet regularly with the course instructors, and participate in a weekly Teaching and Learning seminar. Interested faculty must complete an online application describing how they plan to transform their curricula to incorporate interactive learning strategies such as group problem-solving, project-based labs, or other collaborative activities. Since its inception in 2008, the program has placed approximately 225 LAs in STEM gateway courses, impacting over 8,300 students. In 2011, the STEP project expanded the LA program to Palomar College as part of the effort to strengthen the CSUSM-Palomar partnership in STEM disciplines. From 2012 to 2014 LA's assigned to either campus attended a common weekly Teaching and Learning Seminar, further enhancing student-to-student 
communication between the campuses.

\section{Physics specific linkages}

While the STEP project activities encompassed all STEM disciplines, the project PI's also initiated Physics-specific linkages between the campuses. These efforts included: i) cross-campus learning assistants, ii) faculty-to-faculty meetings focused on the use of technology in the classroom, iii) physics-specific peer-to-peer outreach efforts focused on increasing community between Palomar and CSUSM physics students.

In 2012 and 2013, the project PIs launched a pilot effort to share physics LAs across institutions. Palomar students who had transferred to CSUSM returned to their former campus to support Palomar physics faculty with the implementation of active learning strategies. In addition to supporting course transformation, these LAs were able to talk with the current Palomar students about the transfer process, their experiences in CSUSM physics courses, and the undergraduate research opportunities available at CSUSM.

Faculty-to-faculty connections in physics largely centered on efforts during late 2011 and early 2012 to increase the awareness of, and implementation of pedagogical technologies in the physics classroom. This included meetings to explore personal response systems and the use of screencasts to flip classrooms. Although data suggest that these meetings did not result in a persistent change in the pedagogical approach in Physics at Palomar College, the meetings did result in the organization of a number of visits by Palomar students to CSUSM for joint "rocket car races" and year-end Sigma Pi Sigma induction ceremonies.

\section{Administrative level partnership}

Coordination of the project required regular interaction between project leaders from each campus. These interactions included the initial investment to write and submit the project proposal along with regular monthly project coordination meetings between Palomar and CSUSM PI's. The project also required the formation of advisory boards including administrators from both campuses that met semi-annually to review the project's progress and make recommendations.

\section{RESEARCH DESIGN AND METHODS}

The project employed a mixed methods research design. Quantitative metrics tracking overall changes in student progress towards STEM and Physics Degrees were collected annually (2011-2015) and measured overall progress toward goals along with providing insight into the effect of independent project elements. These metrics included growth of STEM and Physics cohorts, STEM student transfers between institutions, and STEM degree graduates. Quantitative metrics were also collected at the course level for those courses most directly impacted by the program elements. These metrics, such as DFW rates (grades of D, F or Withdrawal) and scores on exams, were used in a quasi-experimental study to compare against prior outcomes in the same courses to identify any differences between the pre-project results and postintervention results.

The project's evaluation protocol also employed qualitative approaches including interviews and surveys of faculty and administrators involved in various project elements. Interviews were conducted by outside project evaluators with faculty and administrators from both campuses. The interviews probed participants' perceptions of the effects of the various project elements and questioned them about changes in institutional nature or approach to STEM and Physics courses during the project.

\section{RESULTS}

Overall results suggest that program activities contributed toward strengthening the partnership between the campuses. Members of both ALPS groups were given a version of the Participant Assessment of Learning Gains survey [8]. This online questionnaire asked the participants to evaluate the extent to which the ALPS activities impacted their professional learning, their access to teaching resources, and their skill development. The results indicate that faculty found the experience to be quite valuable, and all participants expressed interest and willingness to participate in future pedagogical working groups. Describing what she noticed from observing the lesson at Palomar as compared to CSUSM, a Palomar faculty member described that there were: "Two main differences: CSUSM students tended to get their phone out or computer out to figure out how to solve the problem. Palomar students seemed really willing to figure it out themselves. But the Palomar students were also more reluctant to ask for help - to a detriment. At CSUSM, we weren't supposed to really help them [the students], but the CSUSM students were asking the observing Palomar faculty questions and for help, the reverse didn't occur. It was amazing to see that many of the things that our students struggle with, their students struggle with also." In particular, faculty valued the collaborative atmosphere of the working groups; the chance to share perspectives and knowledge with colleagues; and the discussions about effective teaching strategies that could enhance students' learning outcomes.

DFW rates and other student outcomes provide possible evidence for the effectiveness of the ALPS program. For instance, pass rates in courses Calculus I courses with similar students taught by participants improved from $43 \%$ in 2010 to $63 \%$ in 2013 . While student survey responses indicate that the curricular modifications and the interaction with the LAs have contributed to improved affect and increased content knowledge in the introductory calculus courses, it is impossi- 


\section{Number of Palomar transfers}

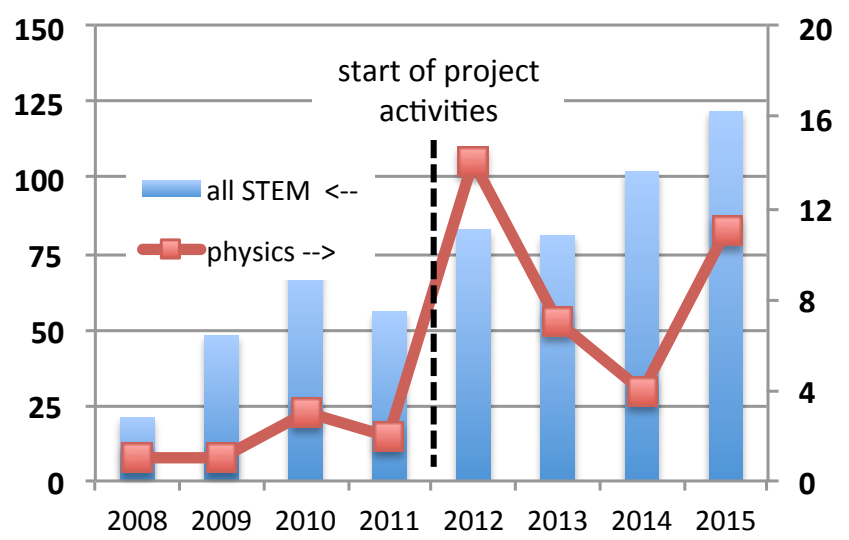

FIG. 1. The number of transfers from Palomar College to CSUSM during project period in all STEM majors (Physics, Biology, Biotechnology, Biochemistry, Chemistry, Mathematics, and Computer Science) and physics specifically.

ble to solely attribute any improvements in passing rates to the ALPS project and the LA program as the greater availability of tutoring and other support initiatives may also have contributed to this outcome. Nonetheless, students appreciate the interactive class format, and they welcome the opportunities to work on problems with peers during class. One participant, a CSUSM adjunct professor who enthusiastically embraced active learning and the LA program philosophy, applied the knowledge he gained from the ALPS discussions to his introductory calculus courses, resulting in significant curricular changes and improved student outcomes. With the support of his LAs, the professor explored various interactive learning strategies including clickers, flipped classrooms, and small group problem-solving.

Interview data suggest that project-supported crosscampus faculty-to-faculty programs such as the ALPS program contributed to a better understanding of the STEM programs at CSUSM. This increased awareness of CSUSM program offerings allowed Palomar faculty to provide higher quality information to prospective transfer students concerning CSUSM programs. As one Palomar faculty member described, "I now have a better understanding of how the math courses work at San Marcos. I have more familiarity with it and a better understanding of how similar their curriculum is to ours. I didn't realize it was that similar. I'm now more able to tell our math majors go over there and get to know the CSUSM faculty - I know that the CSUSM faculty would love that, to have our students go over there. Now I feel I can email them and say, 'I have this student coming over there and they might be a good fit for or interested in a tutoring position...' I could help convey to our students that it will be highly similar when they get there and point out where there might be some subtle differences and to help smooth their transition." Lastly, while it is challenging to isolate the effects of independent course elements, the effects of the program elements as a whole have likely contributed to significant increase in collaboration between the campuses. Results can be seen in the increase of STEM transfers between institutions (Fig. 1).

\section{CONCLUSIONS}

Efforts to establish a robust, reciprocal partnership between a two- and four-year institution led to increases in the number of STEM and physics transfer students and contributed to increases in student success in gateway courses. The crosscampus faculty learning community was valued by faculty for its focus on pedagogy, while also having value in exposing faculty to curriculum and practices on the partner campus. The increase in transfers was likely initiated by administrative linkages, but results suggest that the cross-campus faculty learning community and LA program had a significant impact in increasing student success and smoothing the student transfer process. Challenges remain in disentangling the measurable effects of various program elements. However, taken as a whole, the program elements presented in this paper provide a possible template for other 2- and 4-year institutions seeking to improve pathways to degrees for their shared set of students.

\section{ACKNOWLEDGMENTS}

This work has been supported in part by the NSF DUE1068477, CSU MSTI, and PhysTEC. We thank the many colleagues involved in these efforts.
[1] National Academies, Barriers and Opportunities for 2-Year and 4-Year STEM Degrees: Systemic Change to Support Students' Diverse Pathways, 2016.

[2] C. Henderson, N. Finklestein, and A. Beach, J. Coll Sci Teach. 18 (2010).

[3] C. Henderson, A. Beach, and N. Finklestein, J. Res. Sci. Ed. 48, 8 (2011).

[4] Miami-Ohio, Developing Faculty and Professional Learning Communities (FLCs): Communities of Practice in Higher Education, 2015. Retrieved January 11, 2015, from http://www.units.miamioh.edu/flc/index.php

[5] J. D. Emerson, F. Mosteller, Ed. Media and Tech. Yearbook, 25 pp. 26-42(2000).

[6] D. Sawada, et al. School Sci. Math. 102, pp. 245-253 (2002).

[7] V. Otero, S. Pollock, and N. Finkelstein. Am. J. Phys. 78, 11 (2010).

[8] K. L. Sirum, and D. Madigan, Biochem. and Mol. Bio. Ed. 38, 3 (2010). 